\title{
Pharyngo-cutaneous fistula formation following total laryngectomy: management, sequelae and outcomes
}

\author{
Thomas Pezier ${ }^{1}$, Johannes Rijken ${ }^{2}$, Bernard Tijink ${ }^{1}$, W. Weibel Braunius ${ }^{3}$, and Remco de \\ Bree $^{4}$ \\ ${ }^{1}$ University Medical Centre Utrecht Department of Head and Neck Surgical Oncology \\ ${ }^{2}$ UMC Utrecht \\ ${ }^{3}$ University Medical Center Utrecht \\ ${ }^{4} \mathrm{VU}$ medical center
}

September 2, 2020

\begin{abstract}
Objective: To describe our experience of dealing with pharyngocutaneous fistulas (PCF) following total laryngectomy. Design: Retrospective cohort Setting: Tertiary referral center Participants: All patients undergoing total laryngectomy at the [removed for blind peer review] over the 10 year period January 2008 to December 2017. Main outcome measures: Incidence of PCF; treatment of PCF (conservative versus operative), closure success rate; time to closure; delay in adjuvant radiotherapy; increased the need of future neopharyngeal dilatations; overall survival. Results: A total of 242 patients (199 males, 43 females) were analysed. One hundred and fifteen patients underwent primary laryngectomy, 102 salvage laryngectomy and 25 functional laryngectomy. Seventy five of $242(31 \%)$ patients had a PCF. This was managed conservatively in $36 / 75$ (48\%) and operatively in $39 / 75(52 \%)$. Hospital length of stay was significantly longer in the PCF group (OR 2.38, p=<0.001). 5 year overall survival was $44 \%$ in the no PCF versus $24 \%$ in the PCF group, OR 1.7, p=0.001. PCF was not correlated with delay to adjuvant radiotherapy or the risk of neopharyngeal dilatation. Conclusions: Despite efforts to identify risk factors and take preventive measures, PCF remains an all to frequent complication of total laryngectomy. PCF leads to increased morbidity and significantly worse overall survival. The increased mortality is not a result of a delay in adjuvant radiotherapy.
\end{abstract}

\section{Key points}

- Pharyngo-cutaneous fistula (PCF) occurred in $31 \%$ of cohort of 242 patients

- $81 \%$ of PCFs ultimately healed

- $52 \%$ of PCFs underwent at least 1 operation

- PCF was not associated with a delay in adjuvant radiotherapy nor increased risk of future dilatation

- $\mathrm{PCF}$ is associated with a significantly worse 5 year overall survival ( $44 \%$ vs $24 \%$, , OR $1.7, \mathrm{p}=0.001)$

\section{Introduction}

Total laryngectomy (TL) is used as a primary treatment for advanced stage laryngeal or hypopharyngeal cancer, as salvage in patients with recurrent disease after failure of organ preserving treatments or as a functional treatment in patients with a dysfunctional larynx ${ }^{1-4}$.

Postoperative complications including pharyngocutaneous fistula (PCF) are common ${ }^{1-5}$ with a recent systematic review ${ }^{6}$ reporting rates of up to $58 \%$. PCF often requires additional surgery, delays oral feeding/voice rehabilitation, decreases quality of life and increases hospital stay and $\operatorname{costs}^{3,7,8}$. Furthermore, PCF may 
delay postoperative (chemo)radiotherapy, thus jeopardizing optimal oncological treatment ${ }^{4,9}$ and potentially lead to increased risk of neo-pharyngeal stricture formation.

Many papers describe risk factors for the occurrence of PCF including prior chemoradiotherapy, hypopharyngeal cancer, extensive pharyngeal resection and reconstruction, neck dissections, low BMI and low skeletal muscle mass (sarcopenia) $)^{4,10,11}$. Far fewer articles deal with the management of PCF and its consequences.

In this article we present our single center experience of 242 patients operated over a 10 year period. We evaluate our management strategy with regards to PCF (conservative versus operative), closure success rate and time to closure. We also evaluate whether PCF led to prolonged hospital stay, a delay in adjuvant radiotherapy, increased the need of future neopharyngeal dilatations, or had an adverse effect on overall survival. A rough estimate of extra cost incurred by PCF patients is also given.

\section{Materials and Methods}

We performed a retrospective cohort study of all patients undergoing total laryngectomy at [blinded] over the 10 year period January 2008 to December 2017. The indication for total laryngectomy was either primary, salvage or functional.

Patients' demographic, staging, treatment and outcome data were collected using electronic patient records. All patients were discussed in our multidisciplinary tumour board and underwent total laryngectomy with or without (partial) pharyngectomy. For patients where the pharynx could not be closed primarily, a variety of flaps were used including pectoralis major with or without skin island, free radial forearm flap (FRFF), anterior lateral thigh flap (ALT), jejunum interposition and gastric pull up. Occasionally a pectoralis major muscle onlay flap (also reported in the literature as an interposition flap) was used to reinforce a primarily closed pharynx.

Postoperatively, all patients were fed via a feeding tube for the first 10 days before undergoing a contrast swallow. If this showed no leaks, the patient would begin with clear fluids and quickly build up to a normal diet. If there was a leak, the patient would be kept nil-by-mouth and a further contrast swallow study organised for the following week (unless a PCF manifested itself in during this period).

For this study, PCFs were defined by their clinical manifestation on the skin and patients who had leakage on the contrast swallow but never manifested a PCF were counted as not having a PCF.

The majority of PCFs occurred shortly after surgery, though some patients were discharged either with a feeding tube or on oral diet only to present with a PCF later. These patients were classified as having a PCF. Still further patients developed a PCF many months later secondary to an intervention such as dilatation of a stricture. These patients were classified as no PCF as the development of a PCF was considered a complication of the second procedure, not of the initial laryngectomy.

In those patients who developed a PCF, initial management was conservative using a variety of wound dressings. For fairly dry PCFs, Eusol (Edinburgh University solution of lime) wound dressings were used. For more productive PCFs either iodine soaked gauze or an alginate dressing. For the most productive a stoma bag had to be used. Occasionally scopolamine patches were used to reduce saliva production.

The indication for operative management of a fistula was up to the lead surgeon's judgement. This depended on many factors including the size of the fistula, its response to conservative therapy, the patient's fitness, and the availability of a suitable method of reconstruction (the favoured reconstruction being a salivary bypass tube and pectoralis major flap).

Outcomes of interest included the number of PCFs, duration of PCF, management of PCF (conservative vs operative) and method of reconstruction of the PCF. Some patients died from complications of the fistula and this was also noted. In the longer term, we looked at whether PCF led to a delay in adjuvant radiotherapy (delay was defined as starting radiotherapy $>6$ weeks after surgery) and whether the PCF correlated need 
for dilatation of a neopharyngeal stenosis during follow up. Lastly, we looked at PCF impact on overall survival and make a rough estimation of the extra costs involved in patients with PCF.

Statistical analyses were performed using SPSSß Statistics 20.0 (IBM, Armonk, NY). Overall survival and hospital length of stay was calculated using the Kaplan-Meier method and Cox-mantel log-rank test for comparison. The chi-squared test or binary logistic regression analysis was used as appropriate for univariate analysis.

\section{Results}

A total of 242 patients (199 males, 43 females) underwent total laryngectomy (TL) with or without (partial) pharyngectomy (TLPP) in the study period. Treatment indication was primary $(n=115)$, salvage $(n=102)$ and functional $(n=25)$. Details of the cohort general characteristics stratified by indication is shown in table 1.

Postoperative course /LOS

Mean average length of hospital stay was 21 days (16 days for patients without PCF and 30 days for patients with a PCF, OR 2.38, $\mathrm{p}=<0.001)$, Figure 1 . Out of 242 patients, $51(21 \%)$ returned to theatre: $39 / 51(76 \%)$ for the treatment of PCF, 9/242 (4\%) for hemostasis, 1/242 (0.4\%) for a chyle leak and 2/234 (0.8\%) for exploration of a free flap (one flap successfully salvaged, the other unfortunately lost (jejunum interposition) giving a free flap failure rate of $1 / 17(4.5 \%)$ ).

Incidence of PCFs

A total of $75 / 242(31 \%)$ patients developed a PCF and these were managed as reported in table 2 . In total, $61 / 75$ (81\%) fistulas were successfully closed, $2 / 75(2.7 \%)$ fistulas were still open at last follow-up (respectively 3 and 29 months following laryngectomy) and 12 patients died with an open PCF. These patients are discussed in more detail below.

Conservative Management of PCF

Initial PCF management was conservative with a feeding tube and a variety of wound dressings including Eusol, alginate dressings and/or iodine soaked gauze. Thirty-six out of $75(48 \%)$ PCFs were treated this way with a success rate of 30/36 (83\%). The remaining 6 patients died with open PCFs. Median time to closure was 41 days (see figure 2 ).

Operative management of PCF

The decision to return to theatre was taken by the lead surgeon and depended on a variety of factors including failure of conservative management and fitness of the patient. For most patients, this was during the same hospital stay as the initial laryngectomy, but for a few patients, the surgical management of the PCF was performed months later.

In 39/75 (52\%) PCFs, the patient underwent at least one operation for their fistula. Twenty-three of 29 patients had one operation, 6 patients had 2 operations, 3 patients had 3 operations, 5 patients had 4 operations, 1 patient had 6 operations and 1 patient had 11 operations for his fistula. Twenty-two of 39 (56\%) patients had a regional flap reconstruction (either pectoralis major or delto-pectoral). Of the operatively managed patients, the PCF healed in 31/39 (79\%). Of the patients managed with regional flap reconstruction for their PCF 14/20 (70\%) healed. Of the patients managed with local operative techniques 17/19 (89\%) of PCFs healed.

Median time to closure in the operatively managed patients was 61 days. Kaplan-Meier analysis of time with fistula vs management (conservative vs operative) showed no significant differences ( $\mathrm{p}=0,119$, see Figure 2).

Salivary bypass tubes 
In patients undergoing operative management of their PCF, a salivary bypass tube was used in $32 / 39$ $(82 \%)$ of operated patients. Of the 7 patients managed without a salivary bypass tube 4 had a regional flap reconstruction and 3 a local closure.

Does PCF lead to delayed adjuvant radiotherapy for primary laryngectomy patients?

Post-operative radiotherapy was indicated in 100/115 primary patients. Postoperative radiotherapy was started $>7$ weeks following operation in 28/100 primary patients. Twelve of 28 patients had a PCF versus $16 / 28$ who had a delay despite no PCF. Binary logistic regression showed no correlation between PCF and delay to radiotherapy $(\mathrm{p}=0.06)$. Further worth noting is that 5 patients started adjuvant radiotherapy with an open PCF and in all these 5 patients the PCF closed during the radiotherapy.

Does PCF lead to increased risk of stricture formation necessitating dilatation?

Inflammation, multiple surgeries and prolonged tube feeding associated with PCF may plausibly lead to increased long-term risk of neopharyngeal stricture formation, potentially necessitating dilatation. In our cohort, a total of 44/242 (18\%) of patients ultimately underwent dilatation during follow-up, 15/44 (34\%) had had a PCF. Once again, binary logistic regression showed no statistically significant correlation between $\mathrm{PCF}$ and need for dilatation $(\mathrm{p}=0.623)$.

Does PCF adversely affect perioperative or long-term survival?

Laryngectomy is a physiologically demanding procedure, often performed in patients with little physiological reserve. In our cohort, there were 3/242 (1.2\%) perioperative deaths (defined as deaths within 30 days of laryngectomy). One 76 year old patient had an injury to his common carotid artery intraoperatively which had to be ligated unfortunately leading to an ultimately fatal ischaemic stroke. A 67 year old patient had a fatal myocardial infarction on the first postoperative day. Finally a 76 year old male had necrosis of his pectoralis flap leading to carotid blow-out 1 month after laryngectomy.

In total 12/75 (16\%) patients died with an open PCF which had never healed since the original laryngectomy. In these patients we considered the PCF a complication of the laryngectomy. (Other patients ultimately died of blow-outs associated with pharyngo-cutaneous fistulas due to either local recurrence or complications of dilatation. As the PCF in these cases was not considered a complication of laryngectomy, they are not described below.)

In $1 / 12$ patients with a persistent fistula, the cause of death was unknown. In $3 / 12$ patients there was a separate cause of death (lung embolus, peritonitis and small cell lung cancer). One patient died from distant metastasis. Three of 12 had complications due to their fistula (the blow-out mentioned above, 2 had spondylitis) but no sign of cancer. The remaining 4 patients had biopsy confirmed local residual disease (which retrospectively was probably the reason their PCF had not healed).

We can therefore say that $3 / 75(4 \%)$ patients with PCF died as a direct result of it without sign of cancer.

When we analyzed long term overall survival comparing patients who developed a post-operative PCF and those who did not, we found a significant difference ( 5 year overall survival $24 \%$ versus $44 \%$ respectively, OR $1.7, \mathrm{p}=0.001$, Figure 3).

Costs associated with PCF formation

A complete analysis of the costs associated with PCF formation is beyond the scope of this article. A proper analysis would have to include not only inpatient costs, but also costs for outpatient care and costs associated with inability to work.

Given the differences in LOS and that a hospital bed in our institution very roughly costs 633 Euros per day we can estimate that a PCF patient costs an extra 8862 euros in bed costs alone. This substantial sum does not even take into account that many PCF patients underwent multiple admissions for the management of their PCF, with extra imaging and surgery (PCF patients underwent an average of $1.1(81 / 75)$ operations compared to patients without PCF). Outside of the hospital, PCF patients also required more intensive 
nursing and tube feeding. Several patients with particularly persistent PCFs even underwent hyperbaric oxygen therapy. Along with delays in returning to work, all these factors vastly increase the costs to the healthcare system in case of PCF.

\section{Discussion}

In line with the literature ${ }^{6,12}$, roughly a third of our developed a PCF with a roughly $50 / 50$ split between conservative and operative management. PCFs successfully closed in $61 / 75$ (81\%) cases with both conservative and operative approaches having a similar success rate. We can assume that smaller fistulas were treated conservatively and larger fistulas surgically and this high closure rate reflects the correct strategy for these PCFs. Three of 75 (4\%) patients died of complications from their PCF without sign of cancer. Furthermore, PCF patients had a poorer overall survival (5 year overall survival PCF $24 \%$ versus $44 \%$ in the no-PCF group, OR 1.7, $\mathrm{p}=0.001)$. PCF does not however seem to delay the start of adjuvant radiotherapy $(\mathrm{p}=0.06)$. Indeed, in 5 patients the PCF was deemed small enough to all adjuvant radiotherapy to begin despite an open PCF. PCF also does not increase the risk of future dilatation.

Median length of hospital stay in our cohort was 14 days. This is somewhat higher than in other reports ${ }^{13}$ and may be due to our protocol of keeping patients until their contrast swallow study at 10 days which then allows supervised initiation of feeding and voice rehabilitation. In any case, length of hospital stay was significantly longer in the PCF group than in the no-PCF group (figure 1) which together with the extra nursing care required, extra imaging studies, extra operations and delayed return to work vastly increase the costs associated with PCF.

Given the mortality and morbidity associated with PCF, many surgeons have suggested strategies to reduce their incidence. Pre-operatively there is unfortunately often little time for "pre-habilitation". Intraoperatively, modifications of technique such as the use of barbed sutures ${ }^{14}$, stapler closure ${ }^{15,16}$, salivary bypass tubes ${ }^{17}$, sealants ${ }^{18}$ or even transoral robotic total laryngectomy ${ }^{19,20}$ have all been suggested. Reinforcing primary pharyngeal closure with a pectoralis major muscle-only overlay has also been suggested ${ }^{21}$. This latter strategy is clearly more invasive and is probably only appropriate in high risk patients, though the number-needed-to treat and the extra morbidities and costs associated with an interposition flap are all areas for further investigation. The extra hour of operative time and morbidity of such an interposition flap takes well outweigh the morbidity associated with dealing with a PCF at a later date. Even if patients do suffer a PCF after interposition, there is also the hope that the PCF will be less severe and could be conservatively managed.

Post-operative techniques to reduce the risk of PCF such as reducing saliva (scopolamine patches, botuline injections, parotid duct ligation $)^{22}$, early oral fluids ${ }^{23}$ and vacuum drains ${ }^{24} /$ endoscopic negative pressure $^{2}$ therapy ${ }^{25}$ all have their proponents though remain controversial.

Unfortunately, when there is such a plethora of varying advice in the literature, it is often because no single management option is particularly more effective than any other. Therefore, it remains unclear what the optimal management strategy for prevention and treatment of PCF is.

\section{Conclusions}

PCF remains a troublesome complication after total laryngectomy. Patients with PCF often have prolonged or multiple hospital admissions with associated higher morbidity and costs. Their overall survival is also significantly worse than patients who do not develop a PCF. The risk factors for PCF are numerous and described elsewhere in the literature, as are potential ways to reduce this risk. PCF however does not seem to lead to a delay to adjuvant radiotherapy, nor to an increased risk of stricture formation. 


\section{References}

1. Van der Putten L, De Bree R, Kuik D, et al. Salvage laryngectomy: oncological and functional outcome. Oral oncology.2011;47(4):296-301.

2. Theunissen EA, Timmermans AJ, Zuur CL, et al. Total laryngectomy for a dysfunctional larynx after (chemo) radiotherapy. Archives of Otolaryngology-Head \& Neck Surgery. 2012;138(6):548-555.

3. Paydarfar JA, Birkmeyer NJ. Complications in head and neck surgery: a meta-analysis of postlaryngectomy pharyngocutaneous fistula.Archives of Otolaryngology-Head 83 Neck Surgery.2006;132(1):67-72.

4. Lansaat L, Van Der Noort V, Bernard SE, et al. Predictive factors for pharyngocutaneous fistulization after total laryngectomy: a Dutch Head and Neck Society audit. European Archives of Oto-Rhino-Laryngology. 2018;275(3):783-794.

5. Goepfert RP, Hutcheson KA, Lewin JS, et al. Complications, hospital length of stay, and readmission after total laryngectomy. Cancer.2017;123(10):1760-1767.

6. Liang J-W, Li Z-D, Li S-C, Fang F-Q, Zhao Y-J, Li Y-G. Pharyngocutaneous fistula after total laryngectomy: a systematic review and meta-analysis of risk factors. Auris Nasus Larynx.2015;42(5):353-359.

7. Hasan Z, Dwivedi R, Gunaratne D, Virk S, Palme C, Riffat F. Systematic review and meta-analysis of the complications of salvage total laryngectomy. European Journal of Surgical Oncology (EJSO).2017;43(1):4251.

8. Busoni M, Deganello A, Gallo O. Pharyngocutaneous fistula following total laryngectomy: analysis of risk factors, prognosis and treatment modalities. Acta Otorhinolaryngologica Italica. 2015;35(6):400.

9. Casasayas M, Sansa A, García-Lorenzo J, et al. Pharyngocutaneous fistula after total laryngectomy: multivariate analysis of risk factors and a severity-based classification proposal. European Archives of OtoRhino-Laryngology. 2019;276(1):143-151.

10. Ganly I, Patel S, Matsuo J, et al. Postoperative complications of salvage total laryngectomy. Cancer: Interdisciplinary International Journal of the American Cancer Society.2005;103(10):2073-2081.

11. Bril SI, Pezier TF, Tijink BM, Janssen LM, Braunius WW, de Bree R. Preoperative low skeletal muscle mass as a risk factor for pharyngocutaneous fistula and decreased overall survival in patients undergoing total laryngectomy. Head $\mathscr{E}$ neck.2019;41(6):1745-1755.

12. Virtaniemi JA, Kumpulainen EJ, Hirvikoski PP, Johansson RT, Kosma VM. The incidence and etiology of postlaryngectomy pharyngocutaneous fistulae. Head $\mathcal{E}$ Neck: Journal for the Sciences and Specialties of the Head and Neck. 2001;23(1):29-33.

13. Shenson JA, Craig JN, Rohde SL. Effect of preoperative counseling on hospital length of stay and readmissions after total laryngectomy. Otolaryngology-Head and Neck Surgery. 2017;156(2):289-298.

14. Elmallah RK, Khlopas A, Faour M, et al. Economic evaluation of different suture closure methods: barbed versus traditional interrupted sutures. Annals of translational medicine. 2017;5(Suppl 3).

15. Calli C, Pinar E, Oncel S. Pharyngocutaneous fistula after total laryngectomy: less common with mechanical stapler closure. Annals of Otology, Rhinology \&3 Laryngology. 2011;120(5):339-344.

16. Aires FT, Dedivitis RA, Castro MAF, Bernardo WM, Cernea CR, Brandao LG. Efficacy of stapler pharyngeal closure after total laryngectomy: a systematic review. Head \& neck. 2014;36(5):739-742.

17. Montgomery WW. Salivary bypass tube. Annals of Otology, Rhinology \& Laryngology. 1978;87(2):159162.

18. Stephenson KA, Pandey S, Lubbe DE, Fagan JJ. Use of surgical sealant in the prevention of pharyngocutaneous fistula after total laryngectomy.Head $\&$ neck. 2018;40(12):2606-2611. 
19. Lawson G, Mendelsohn A, Fakhoury R, et al. Transoral Robotic Surgery Total Laryngectomy. ORL. 2018;80(3-4):171-177.

20. Krishnan G, Krishnan S. Transoral robotic surgery total laryngectomy: evaluation of functional and survival outcomes in a retrospective case series at a single institution. ORL.2017;79(4):191-201.

21. Guimarães AV, Aires FT, Dedivitis RA, et al. Efficacy of pectoralis major muscle flap for pharyngocutaneous fistula prevention in salvage total laryngectomy: a systematic review. Head \& neck.2016;38(S1):E2317E2321.

22. Guntinas-Lichius O, Eckel HE. Temporary reduction of salivation in laryngectomy patients with pharyngocutaneous fistulas by botulinum toxin A injection. The Laryngoscope. 2002;112(1):187-189.

23. Timmermans AJ, Lansaat L, Kroon GV, Hamming-Vrieze O, Hilgers FJ, van den Brekel MW. Early oral intake after total laryngectomy does not increase pharyngocutaneous fistulization. European Archives of Oto-Rhino-Laryngology. 2014;271(2):353-358.

24. Roberts ST, Hobson M, Eisenberg R. A simple drainage technique to manage pharyngocutaneous fistula following laryngectomy. Clinical Otolaryngology. 2019;44(6):1211-1213.

25. Loeck J, von Lucken H-J, Kehrl W, Loske G. Endoscopic negative pressure therapy (ENPT) of a postlaryngectomy pharyngocutaneous fistula: first report of a new treatment method. HNO.2019;67(2):77-79.

\section{Hosted file}

Tables.docx available at https://authorea.com/users/354924/articles/478388-pharyngocutaneous-fistula-formation-following-total-laryngectomy-management-sequelae-andoutcomes

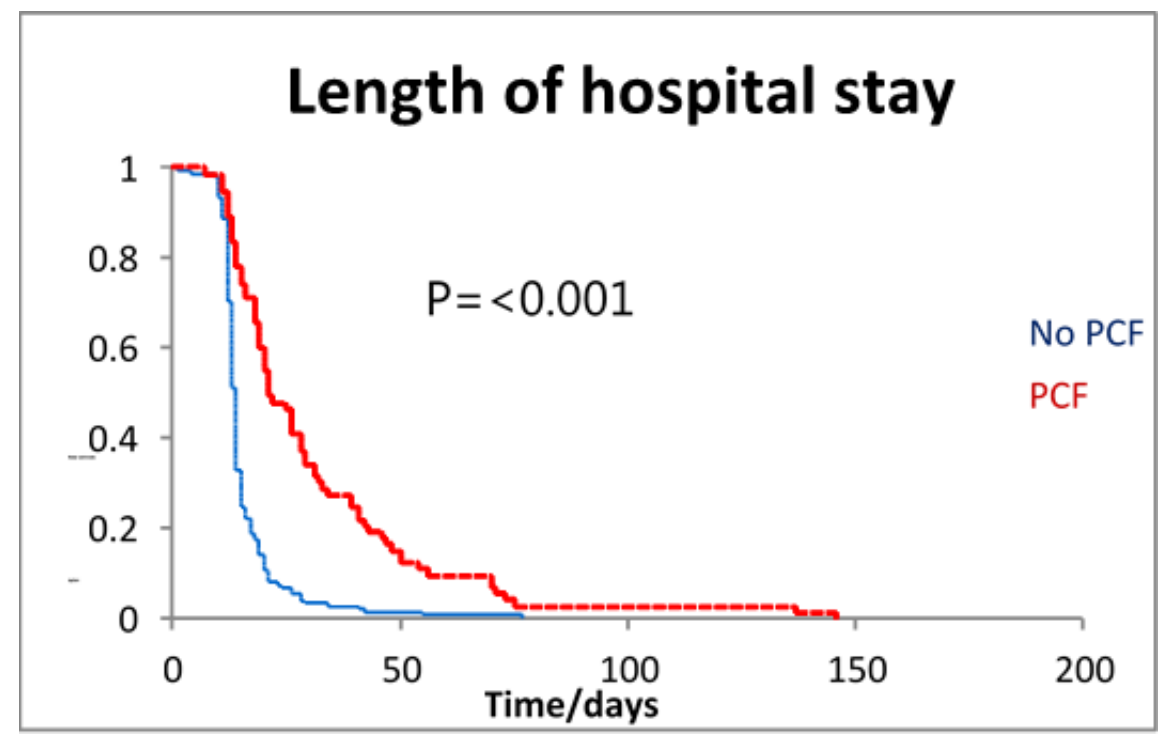

\section{Figure 1: Length of hospital stay}




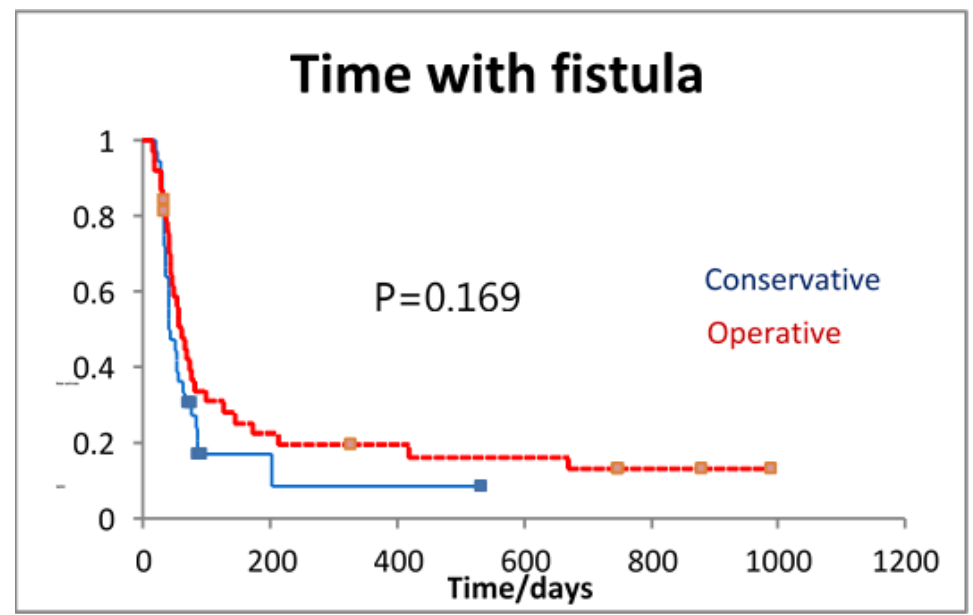

Figure 2: Time with fistula, conservative vs operative management.

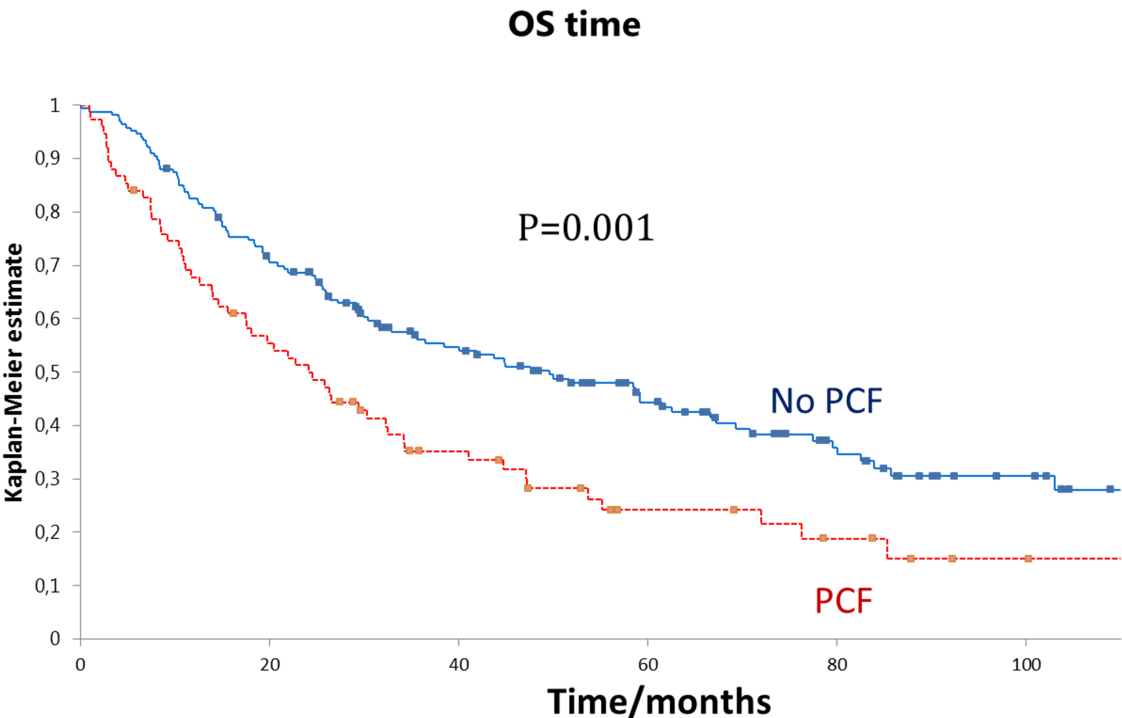

Figure 3: Kaplan Meier analysis of overall survival. 